\title{
Economic burden of antibiotic resistance in ESKAPE organisms: a systematic review
}

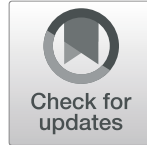

Xuemei Zhen ${ }^{1,2}$, Cecilia Stålsby Lundborg ${ }^{2}$, Xueshan Sun ${ }^{1}$, Xiaoqian $\mathrm{Hu}^{1}$ and Hengjin Dong ${ }^{1,3^{*}}$ (D)

\begin{abstract}
Background: Antibiotic resistance (ABR) is one of the biggest threats to global health. Infections by ESKAPE (Enterococcus, S. aureus, K. pneumoniae, A. baumannii, P. aeruginosa, and E. coli) organisms are the leading cause of healthcare-acquired infections worldwide. ABR in ESKAPE organisms is usually associated with significant higher morbidity, mortality, as well as economic burden. Directing attention towards the ESKAPE organisms can help us to better combat the wide challenge of ABR, especially multi-drug resistance (MDR).

Objective: This study aims to systematically review and evaluate the evidence of the economic consequences of ABR or MDR ESKAPE organisms compared with susceptible cases or control patients without infection/colonization in order to determine the impact of ABR on economic burden.
\end{abstract}

Methods: Both English-language databases and Chinese-language databases up to 16 January, 2019 were searched to identify relevant studies assessing the economic burden of ABR. Studies reported hospital costs (charges) or antibiotic cost during the entire hospitalization and during the period before/after culture among patients with ABR or MDR ESKAPE organisms were included. The costs were converted into 2015 United States Dollars. Disagreements were resolved by a third reviewer.

Results: Of 13,693 studies identified, 83 eligible studies were included in our review. The most studied organism was S. aureus, followed by Enterococcus, A. baumannii, E. coli, E. coli or/and K. pneumoniae, P. aeruginosa, and K. pneumoniae. There were 71 studies on total hospital cost or charge, 12 on antibiotic cost, 11 on hospital cost or charge after culture, 4 on ICU cost, 2 on hospital cost or charge before culture, and 2 on total direct and indirect cost. In general, ABR or MDR ESKAPE organisms are significantly associated with higher economic burden than those with susceptible organisms or those without infection or colonization. Nonetheless, there were no differences in a few studies between the two groups on total hospital cost or charge (16 studies), antibiotic cost (one study), hospital cost before culture (one study), hospital cost after culture (one study). Even, one reported that costs associated with MSSA infection were higher than the costs for similar MRSA cases.

Conclusions: ABR in ESKAPE organisms is not always, but usually, associated with significantly higher economic burden. The results without significant differences may lack statistical power to detect a significant association. In addition, study design which controls for severity of illness and same empirical antibiotic therapy in the two groups would be expected to bias the study towards a similar, even negative result. The review also highlights key areas where further research is needed.

Keywords: Antibiotic resistance, Economic burden, ESKAPE organism, S. aureus, Enterococcus, A. baumannii, E. coli, K. pneumoniae, P. aeruginosa

\footnotetext{
* Correspondence: donghj@zju.edu.cn

${ }^{1}$ Center for Health Policy Studies, School of Public Health, Zhejiang University

School of Medicine, 866 Yuhangtang Road, Hangzhou 310058, Zhejiang,

China

${ }^{3}$ The Fourth Affiliated Hospital Zhejiang University School of Medicine, No.

N1, Shancheng Avenue, Yiwu City, Zhejiang, China

Full list of author information is available at the end of the article
}

(c) The Author(s). 2019 Open Access This article is distributed under the terms of the Creative Commons Attribution 4.0 International License (http://creativecommons.org/licenses/by/4.0/), which permits unrestricted use, distribution, and

reproduction in any medium, provided you give appropriate credit to the original author(s) and the source, provide a link to the Creative Commons license, and indicate if changes were made. The Creative Commons Public Domain Dedication waiver (http://creativecommons.org/publicdomain/zero/1.0/) applies to the data made available in this article, unless otherwise stated. 


\section{Background}

Antibiotics have been pivotal in treating and preventing common infections, but the laws of evolution and natural selection along with the overuse and misuse have contributed to an alarming increase in antibiotic resistance (ABR) worldwide. As the selection of antibiotics is getting smaller together with only slow changes in prescription behavior, we are heading for a post-antibiotic era [1]. ABR is one of the biggest threats to global health, endangering not only the achievements towards the Millennium Development Goals but also the Sustainable Development Goals [2]. ABR is usually associated with significant higher morbidity, mortality, prolongation of illness and reduced labour efficiency [3-9]. In high-income countries, it was estimated that ABR resulted in as much as $\$ 20$ billion in excess direct costs, with $\$ 35$ billion in societal costs for lost productivity each year in the United States (US) alone [10]. In the European Union (EU) and European Economic Area (EEA) countries, a subset of ABR organisms is associated with extra healthcare costs and lost productivity amounting to $€ 1.1-1.5$ billion yearly if there is no prompt and effective action [11, 12]. Globally, it would lose $1.1-3.8 \%$ of its annual gross domestic product (GDP) due to antimicrobial resistance (AMR) by 2050 [13]. Low- and middle-income countries will suffer more [14]. Countries in the sub-Saharan Africa may face a GDP loss of 0.1-2.5\% [14]. A total of 24 million people would be forced into extreme poverty due to AMR by 2030 , especially in low-income countries [13].

To combat ABR, the World Health Organization (WHO) in 2015 published a global action plan, it is expected that individual countries will develop their own national action plans on AMR in keeping with this global plan. However, the absence of economic assessments on economic burden of ABR is an obstacle to implementation of global or national strategies for containment of $A B R$ [15]. It is necessary to conduct economic research to assess the costs of $A B R$ and the costs and benefits of global, national or regional action plans [15].

The WHO also developed a global priority list of ABR organisms to guide the research, discovery, and development of new antibiotics [16]. In this list, Enterococcus spp., Staphylococcus aureus, Klebsiella pneumoniae, Acinetobacter baumannii, Pseudomonas aeruginosa, and Escherichia coli, collectively termed ESKAPE, have been identified as being increasingly involved in infectious diseases in humans. There were reports of third-generation cephalosporins resistance and fluoroquinolone resistance in E. coli exceeding $50 \%$ in five out of six WHO regions (African region, region of the Americas, Eastern Mediterranean region, European region, South-East Asia region, and Western Pacific region). For K. pneumonia, the six WHO regions had more than $50 \%$ resistance to third-generation cephalosporins and two WHO regions had more than $50 \%$ resistance to carbapenems. The overall Methicillin resistant $S$. aureus (MRSA) proportions exceeded $20 \%$ in the six WHO regions, and even exceeded $80 \%$ in three WHO regions [17].

ESKAPE organisms are the leading cause of healthcare-associated infections all over the world [18], especially in critically ill and immunocompromised individuals. These organisms consistently "escape" the effects of commonly used antibiotics and are a critical threat to public health [19]. Focusing attention on these pathogenic organisms is important since some studies have shown that patients with ABR ESKAPE organisms are more likely to receive inappropriate antibiotic therapy resulting in higher mortality rates and opportunities for spreading to other patients [20-23].

Several studies have examined the economic outcomes of resistant ESKAPE organisms in general and multidrug resistance (MDR) specifically, but there has not been an in-depth, comparative analysis of the contemporary literature reporting on costs associated with resistant versus susceptible cases. In this study, we aimed to analyze the published literature of the economic consequences of resistant or MDR ESKAPE organisms compared with susceptible cases or control patients without infection/colonization.

\section{Methods}

\section{Literature search}

We performed a systematic search in the English-language databases (PubMed, Web of Science, and Embase) and Chinese-language databases (China National Knowledge Infrastructure, Wanfang data, and Chongqing VIP) up to January 16, 2019. In addition, we also manually reviewed the references from retrieved studies to ensure inclusion of all published studies. Detailed search strategies are provided in Additional file 1.

\section{Study selection}

Inclusion and exclusion criteria were predefined. Inclusion criteria included (1) studies published in English or Chinese language; (2) publication date between January 1, 2000 and January 16, 2019; (3) original research of any type (cohort, case control, or observational study); (4) reports on humans; (5) reports on ESKAPE organisms; (6) reports on resistant versus susceptible cases or those without infection or colonization; and (7) reports on economic burden. Studies published before 2000 were not considered to ensure that the analysis focuses on contemporary literature that reflects current resistance patterns and clinical practice guidelines [4, 5]. Studies reporting on a group organisms (e.g. Gram-positive organisms, Gram-negative organisms, Enterobacteriaceae, Enterobacter species, etc.) were excluded as well. Both E. coli and K. pneumonia are members of 
Enterobacteriaceae, sharing characteristics and were therefor analyzed together. Two reviewers independently evaluated studies for eligibility based on titles and abstracts, then, reviewed the full text to decide if it met the inclusion criteria. Disagreements were resolved by a third reviewer.

\section{Data extraction}

We developed a standardized extraction form to record the characteristics of each study, including first author, publication year, type of study, method, country, study setting, study period, study population, type of infection, type of hospital ward, organisms, sample size (cases and controls). Regarding the costs, population-adjusted costs were showed because that susceptible rates are more frequent than resistant rate, thus, susceptible organisms will cause more infections than resistant ones, in general. We extracted the currency and cost year, total hospital costs (charges) or antibiotic cost in median or mean values, and the statistical analysis of the cost differences. Costs were converted into 2015 US dollars using average exchange rates, then inflating this to 2015 currency estimates using the annual consumer price index $[24,25]$. Hospital charge was defined as the amount that patient is expected to pay for care. Hospital cost was defined as expenses incurred by a hospital in providing patient care, including the sum of hospital charges and the amount from the reimbursement service. Again, disagreements were resolved by the third reviewer.

\section{Study quality assessment}

The Newcastle-Ottawa quality assessment Scale (NOS) for cohort and case-control studies was used to assess study quality. A "star system" was developed to judge the study on three broad perspectives: (1) selection population (four items); (2) comparability of the groups (one item); and (3) ascertainment of either the exposure or outcome of interest (three items). The highest study quality in NOS was nine "stars" where $\geq 7$ stars indicated high-quality studies, 4-6 stars as moderate and $\leq 3$ stars as low quality [3-5] (Additional file 2).

\section{Results}

\section{Studies identified}

A total of 13,693 relevant studies were identified by original database searching. Seven additional studies were identified through other sources. Based on review of titles only, 8930 studies were retrieved after excluding duplicates. Abstract screening resulted in 351 papers for detailed full-text assessment based on the same criteria. Eighty-three studies were finally eligible for this systematic review (Fig.1).

\section{Study characteristics and quality}

Of the 83 eligible studies included in our review, ten were prospective observational studies, and 73 were retrospective studies. Twenty-one studies were conducted in multiple hospital settings. The countries with the largest number of studies were the US $(n=40)$, followed by China $(n=16$; mainland, China: $n=12$; Taiwan, China: $n=4)$, Germany $(n=6)$, Thailand $(n=5)$, South Korea $(n=4)$, Canada $(n=3)$, Spain $(n=3)$, Australia $(n=1)$, Colombia $(n=1)$, Italy $(n=1)$, Mexico $(n=1)$, the United Kingdom $(n=1)$, and EU and EEA (31 countries) $(n=1)$. The most studied organism was $S$. aureus $(n=32)$, followed by Enterococcus $(n=16), A$. baumannii $(n=12)$, E. coli $(n=11), E$. coli or/and $K$. pneumoniae $(n=8), P$. aeruginosa $(n=8)$, and $K$. pneumoniae $(n=3)$. Nine studies reported the economic outcome of ABR in ESKAPE organisms in the intensive care unit (ICU). Five studies included only colonized patients, 17 for hospital-acquired infection, and seven for community-acquired infection. For the sources of infection, 21 studies were bacteremia or septicemia or bloodstream infection (BSI), seven were pneumonia, four were urinary tract infection (UTI), two were surgical site infection, two were skin and soft tissue infection, and two were intra-abdominal infection (IAI) (Additional file 3: Table S1-S7).

For the cost, there were 71 studies on total hospital cost or charge, 12 on antibiotic cost, 11 on hospital cost or charge after culture, 4 on ICU cost, 2 on hospital cost or charge before culture, and 2 on total direct and indirect cost. Most of studies reported that ABR or MDR ESKAPE organisms were significantly associated with higher hospital costs than those with susceptible organisms or those without infection or colonization. Nonetheless, there were no differences in a few studies between the two groups on total hospital cost or charge (16 studies), antibiotic cost (one study), hospital cost before culture (one study), hospital cost after culture (one study). Even, one reported that costs associated with MSSA infection were higher than the costs for similar MRSA cases [26] (Tables 1, 2, 3, 4, 5, 6 and 7). Propensity score matching, simply matching, multivariate linear regression model, generalized linear model, and significant test were the most utilized methods (Additional file 3: Table S1-S7). We judged 66 were high quality studies and 17 were of moderate quality (Additional file 2).

\section{Staphylococcus aureus}

For $S$. aureus, the control groups were categorized into two groups namely methicillin susceptible $S$. aureus (MSSA) and non-infection. Mean or median total hospital cost or charge among inpatients with MRSA was 1.12 times-6.25 times higher than that for MSSA hospitalizations [37-47, 51]. The median cost difference 

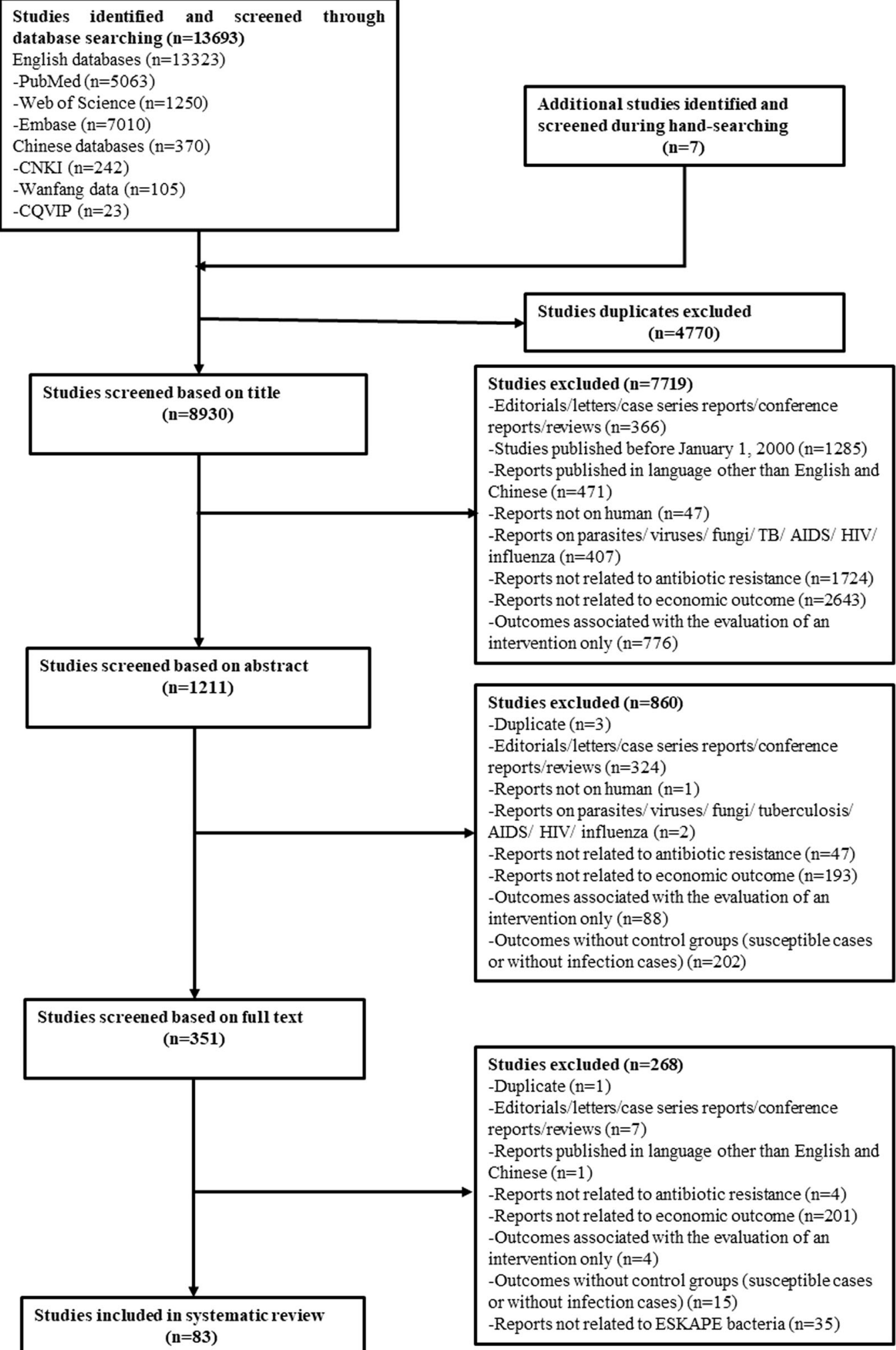

Fig. 1 Flowchart of literature search. CNKI China National Knowledge Infrastructure, CQVIP Chongqing VIP, AIDS acquired immunodefiency syndrome, HIV human immunodefienccy virus, ESKAPE Enterococcus spp, Staphylococcus aureus, Klebsiella pneumoniae, Acientobacter baumannii, Pseudomonas aeruginosa, and Escherichia coli 


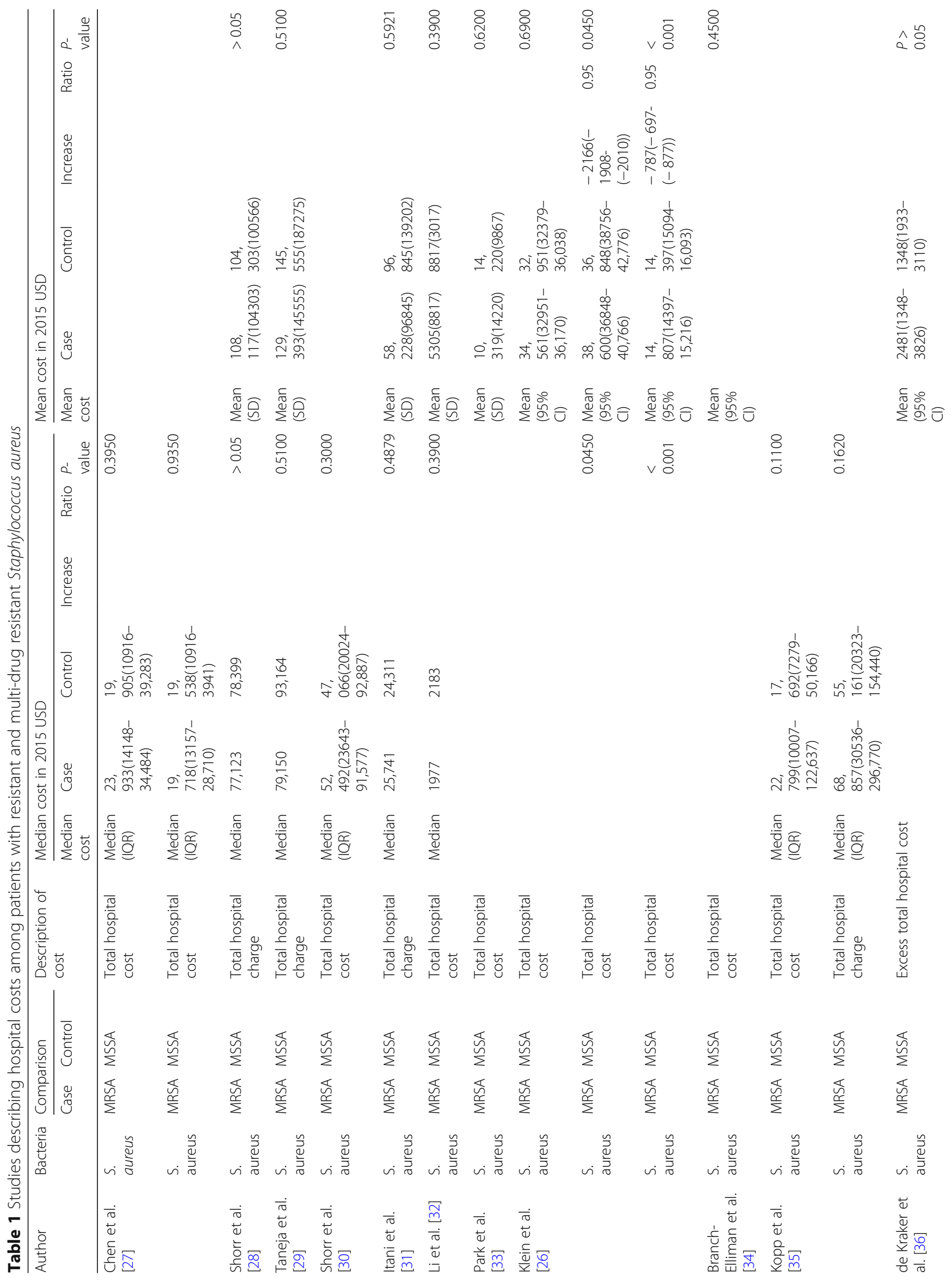




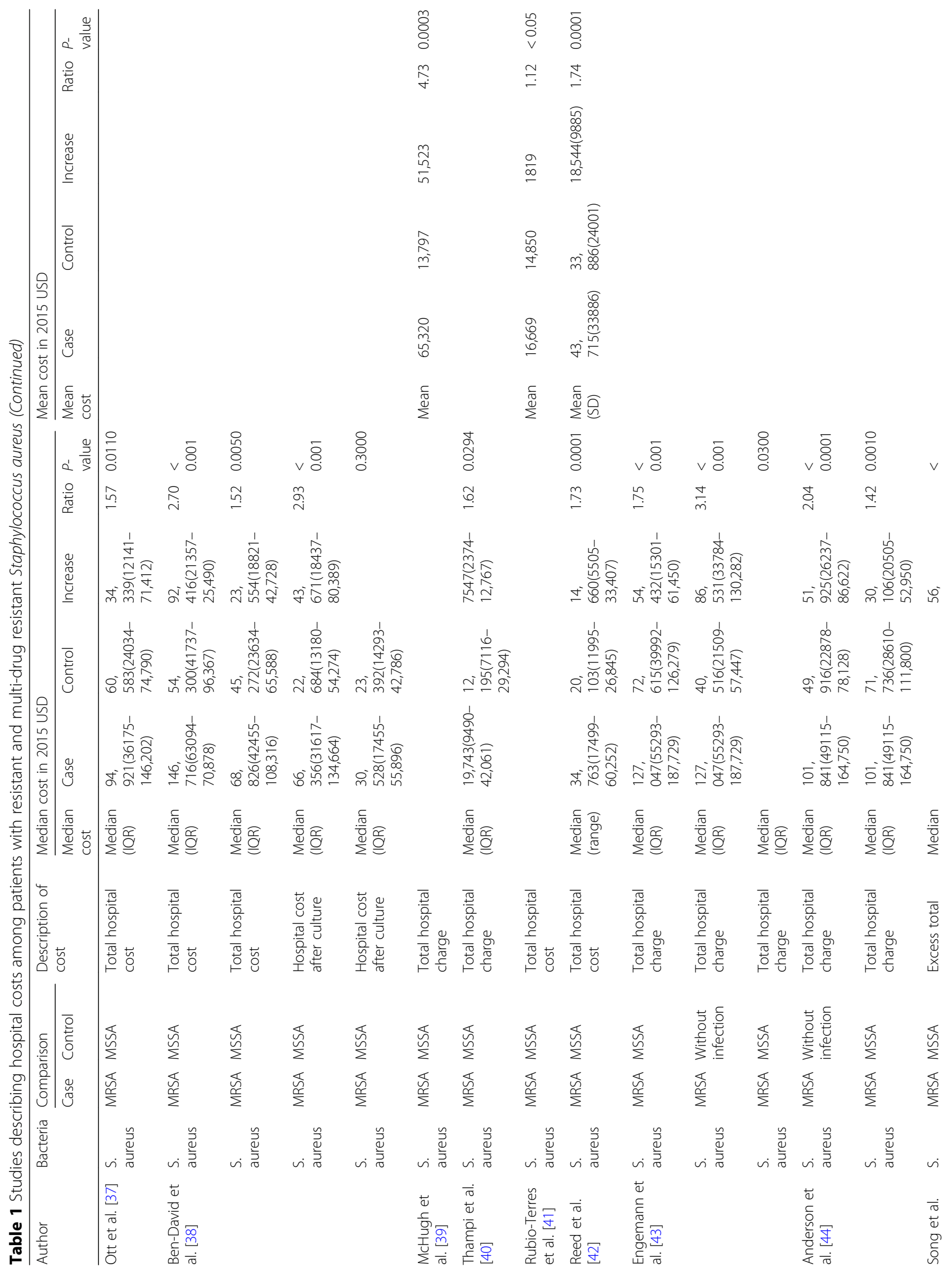




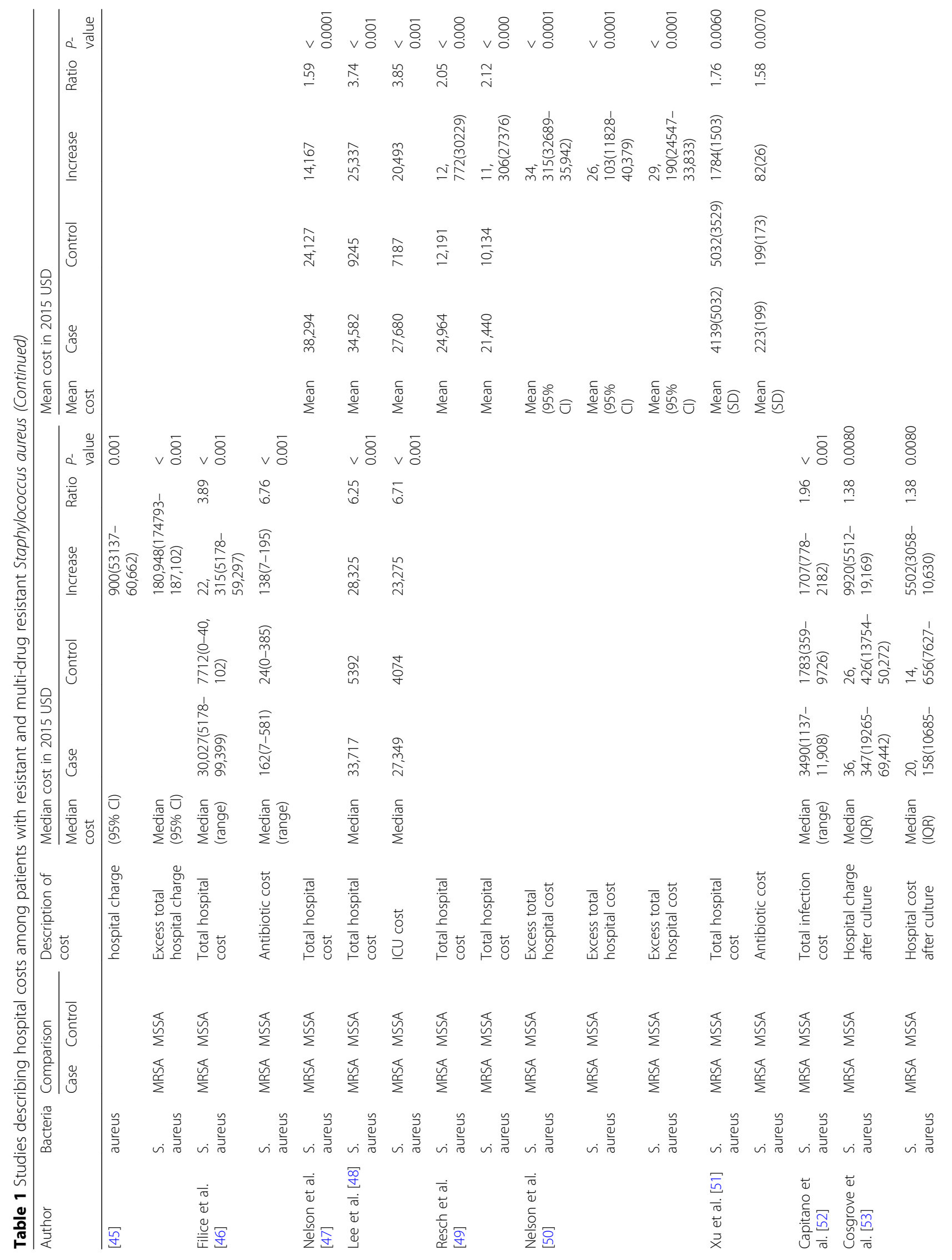




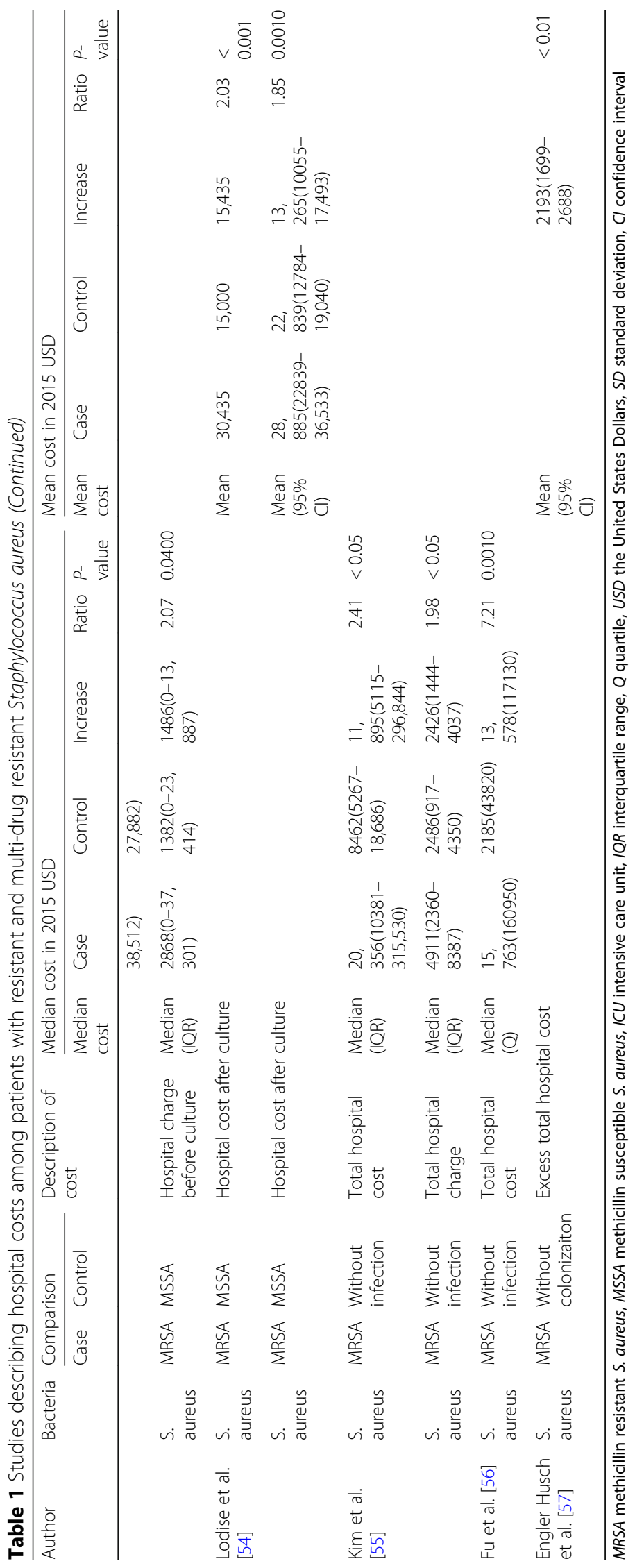




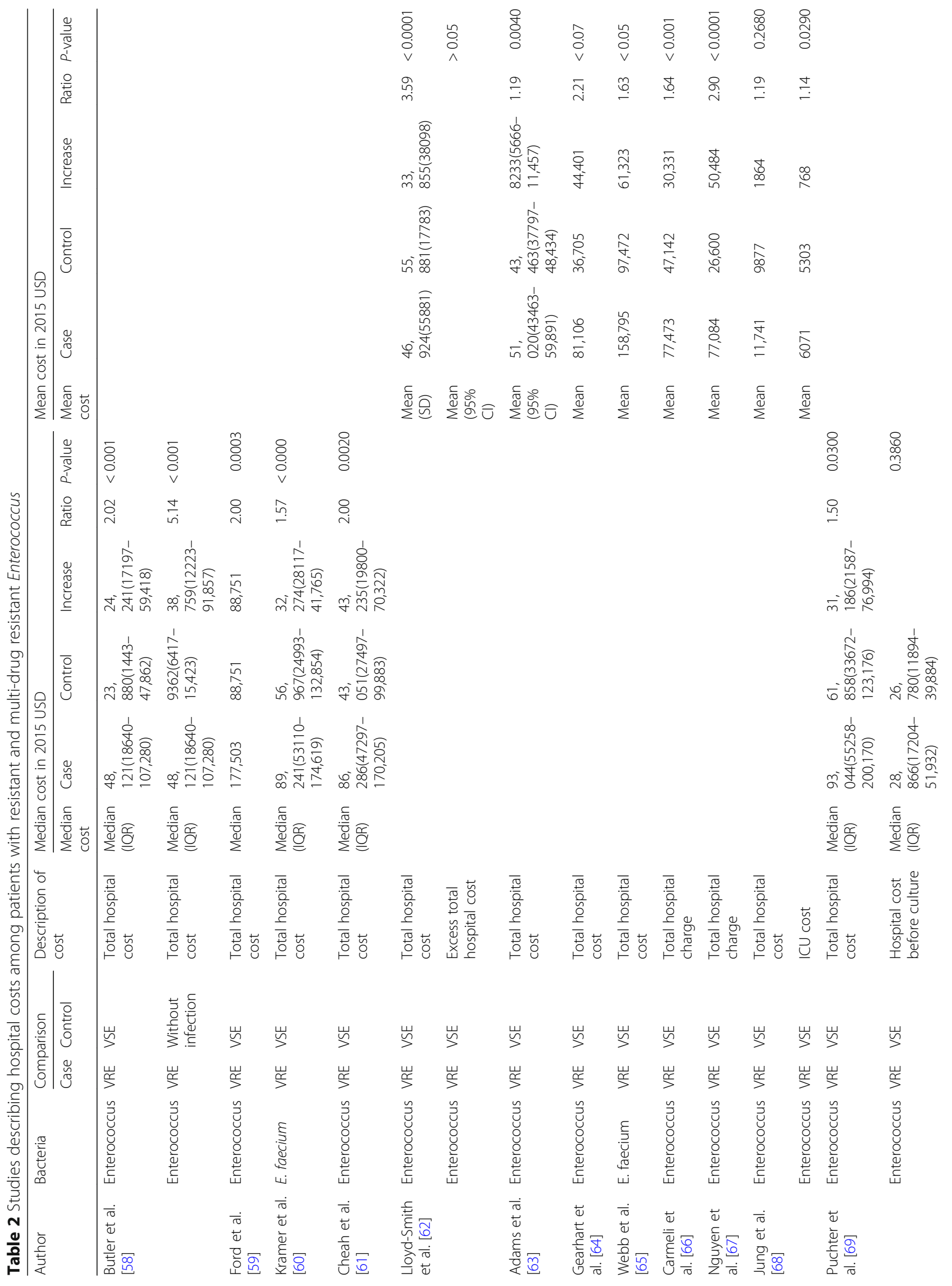




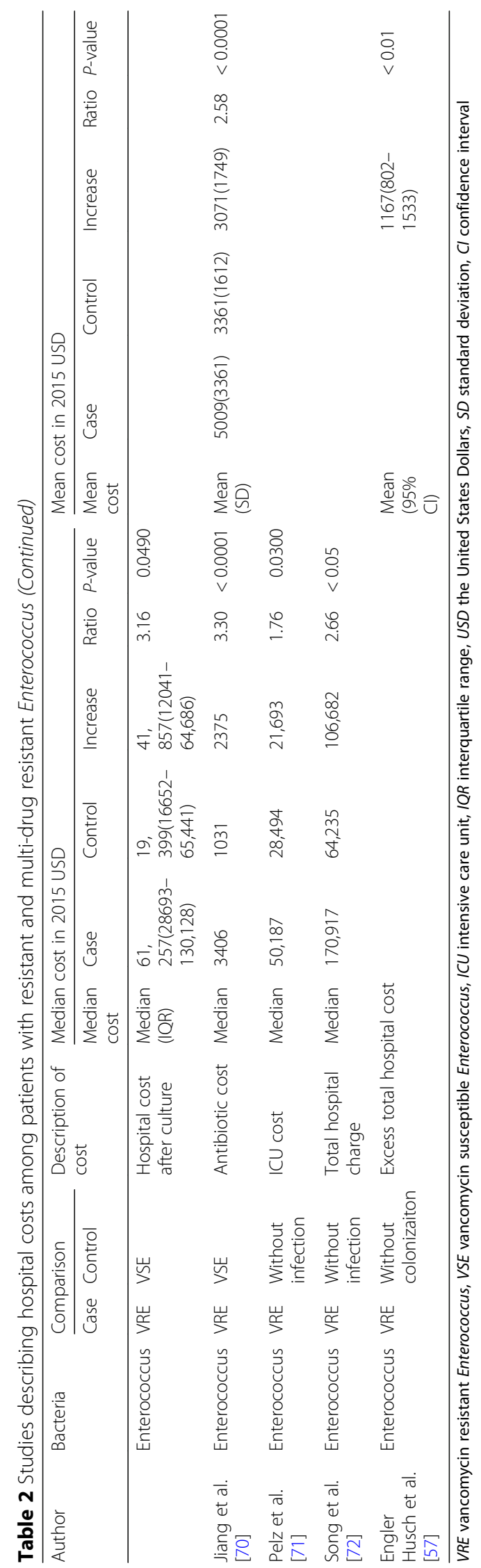




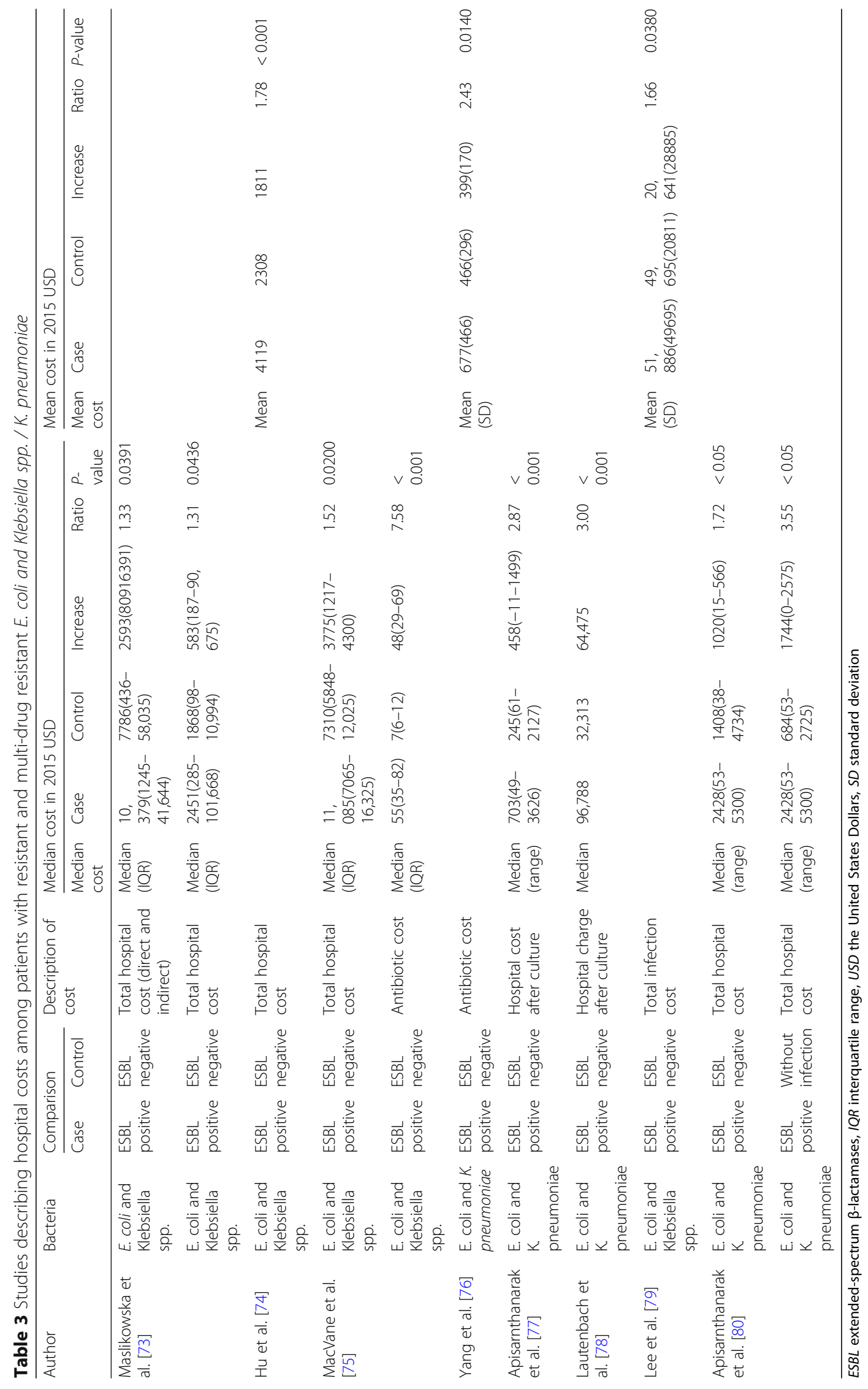




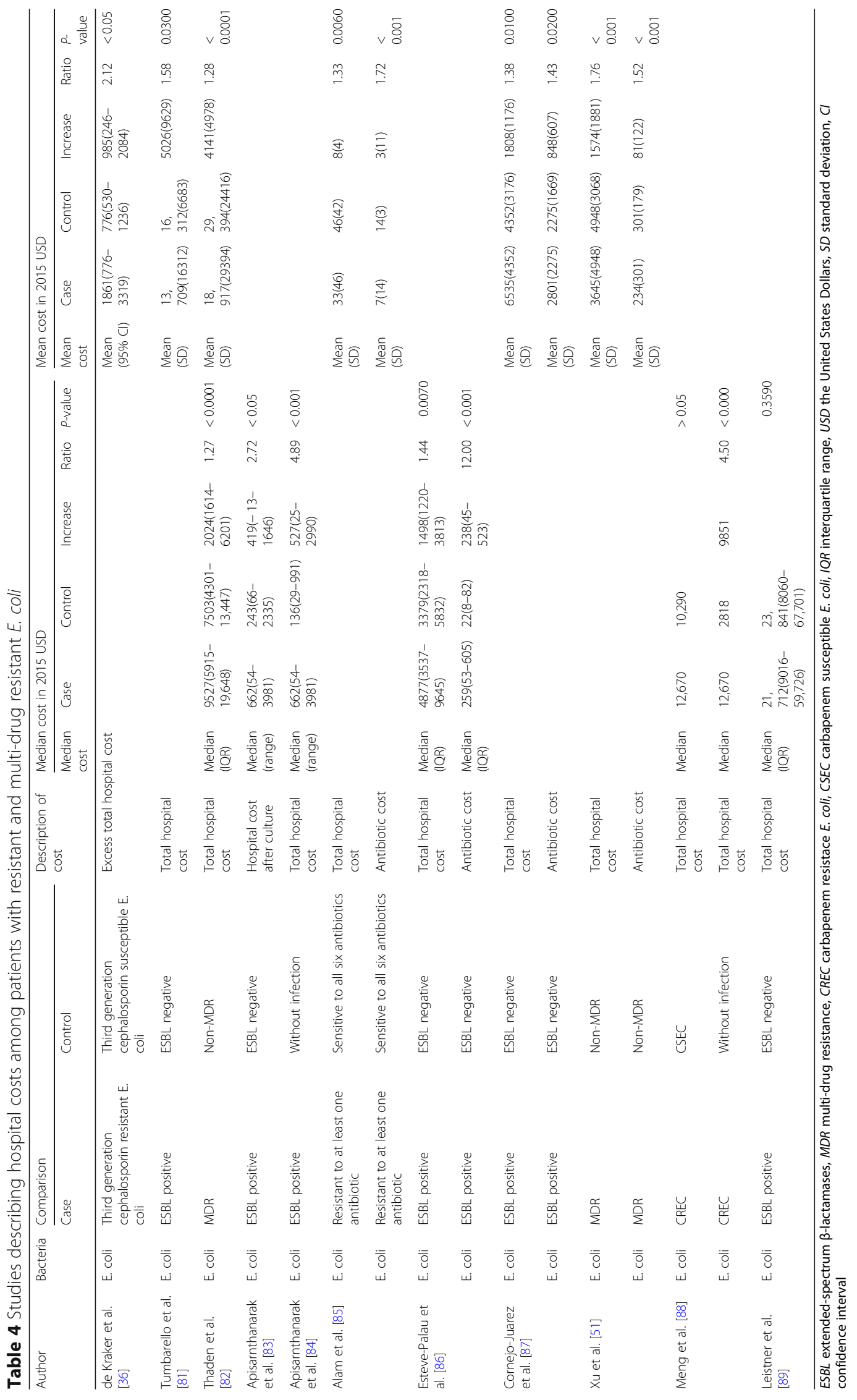




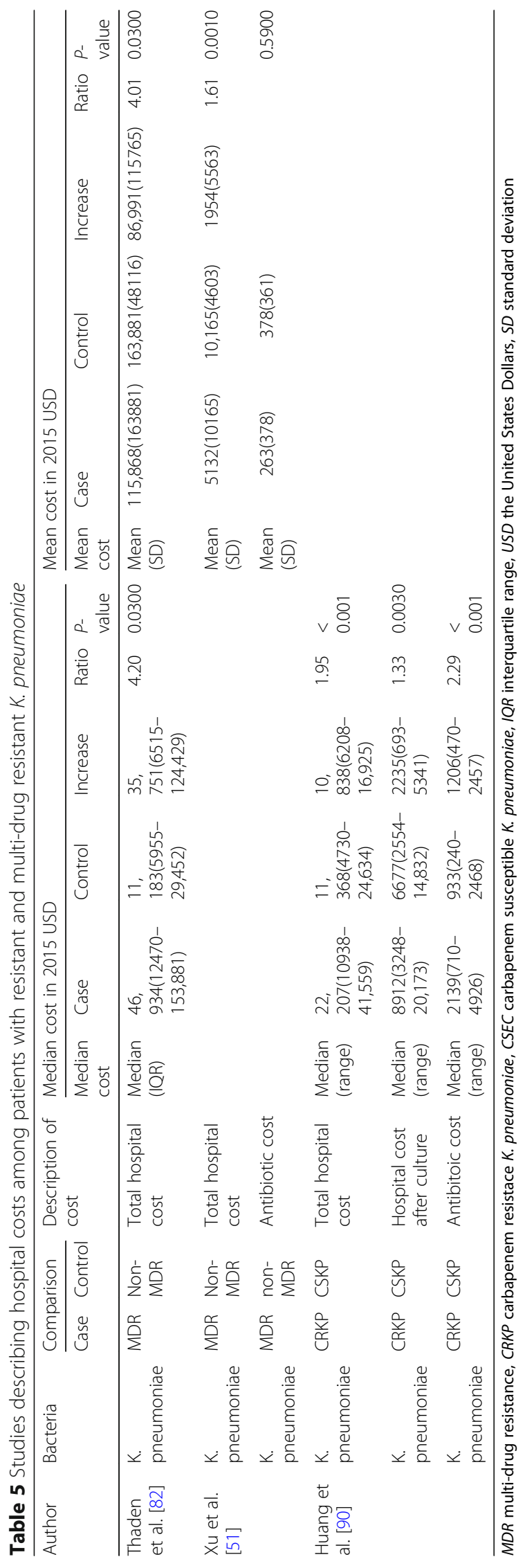




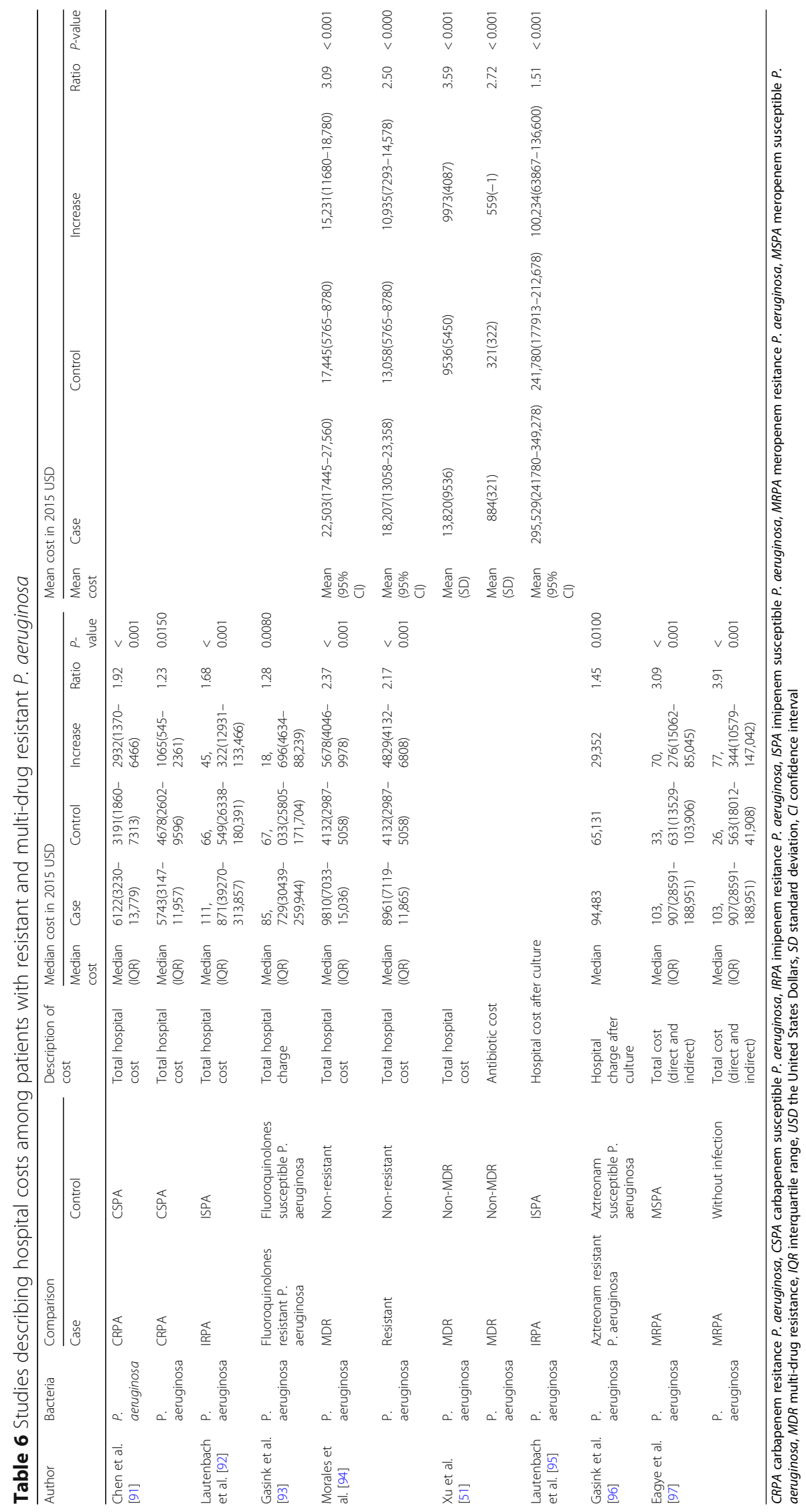




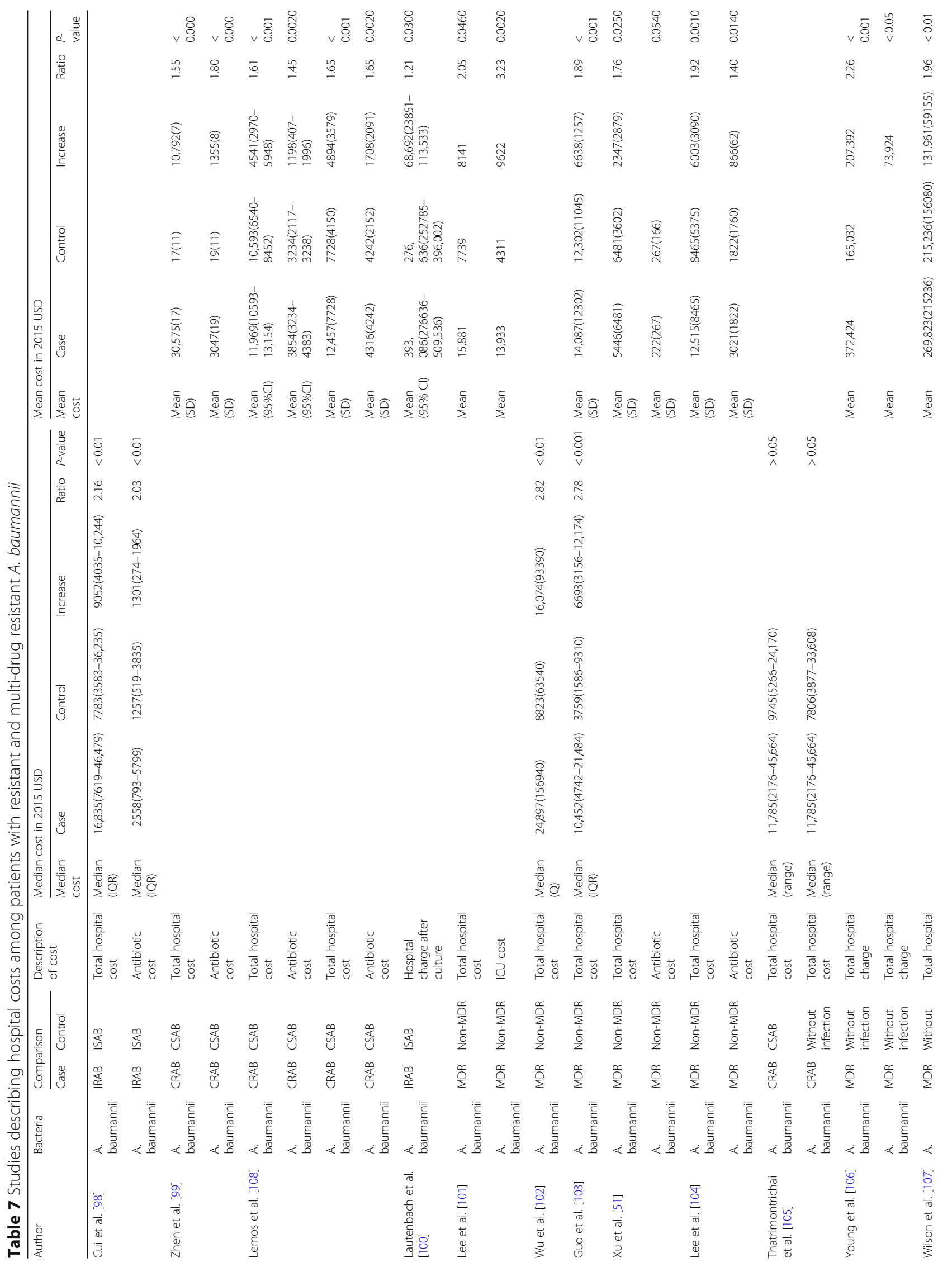




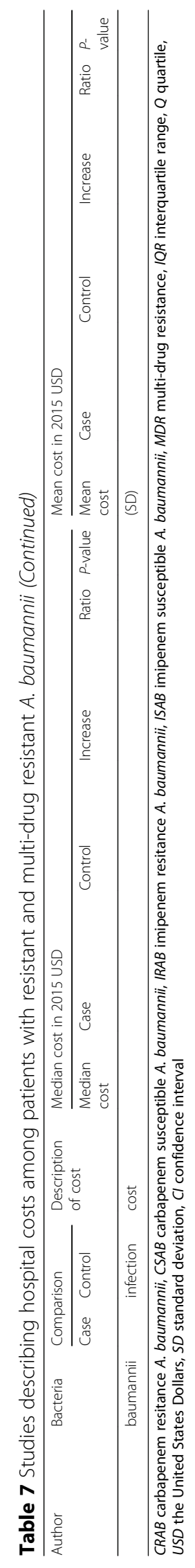


ranged from $\$ 7547$ in Canada [40] to $\$ 180,948$ in the US [45], and the mean cost difference ranged from $\$ 1784$ in China [51] to $\$ 51,523$ in the US [39]. Median cost in total infection cost, ICU cost, and hospital charge before culture among inpatients with MRSA were 1.96 times [52], 6.71 times [48], and 2.07 times [53] as high as that for MSSA cases, respectively. Compared with inpatients with MSSA, MRSA cases were associated with ranging from 1.58 times [51] to 6.75 times [46] of mean or median antibiotic cost, and ranging from 1.38 times [53] to 2.93 times [38] of mean or median hospital cost or charge after culture. However, there were no differences in total hospital cost or charge in 11 studies [26-35, 60] and in hospital cost after culture in one study [38] between the two groups.

In addition, compared with inpatients without infection, those with MRSA were associated with 1.98 times-7.21 times higher total hospital cost or charge $[44,55,56]$. ICU is a significant driver behind increased hospital costs, MRSA in the ICU was associated with four times higher additional total hospital cost than that in general ward [38]. Type of infection is also an important factor of hospital costs [38]. Compared with inpatients with MSSA BSI, surgical site infection, and pneumonia, those with MRSA were significantly associated with 52$170 \%$ [38-42, 55], $75-214 \%$ [43, 44, 68], and $57 \%$ [37] of increased median total hospital cost or charge, respectively. $12-373 \%[39,41,42]$ and $78 \%$ [74] of increased mean total hospital cost or charge were incurred among inpatients with MRSA BSI and IAI than those with MSSA, respectively. Nevertheless, it is showed that there were no differences in total hospital cost or charge among BSI [26, 33, 36], breast abscess [34], skin and soft tissue infection [31, 32, 34, 72], and pneumonia [27-30] between the two groups. There was an additional median total hospital charge of $\$ 56,900$ [45] and mean total hospital cost of \$ 2193 [57] for MRSA colonization than that for MSSA colonization and those without colonization, respectively.

Contrary to the historical studies at the hospital level, Klein et al. found that costs associated with MSSA-related infection were similar with and even surpass costs of MRSA-related infections [26] (Table 1 and Additional file 3: Table S1 and S8 ).

\section{Enterococcus}

For Enterococcus, the control groups were also divided into two groups namely vancomycin susceptible Enterococcus (VSE) and non-infection. Median total hospital cost among inpatients with VRE BSI was 1.57-2.02 times higher than that for VSE cases [58-61], with the increased cost ranging from $\$ 24,241$ [58] and $\$ 88,751$ in the US [59]. In addition, inpatients with VRE were associated with $19-259 \%$ of increased mean total hospital cost or charge than those with VSE [62-67], ranging from $\$ 8233$ [63] to $\$ 61,323$ [65]. However, some studies reported that there were no differences in total hospital cost $[62,68]$ or hospital cost before culture [69].

Median costs in antibiotic cost and hospital cost after culture among inpatients with VRE were 3.30 times [70] and 3.16 times [69] as high as that for VSE cases, respectively. VRE colonization was found not to be a significant factor associated with total hospital cost, nonetheless, it resulted in a significant increase in ICU cost after controlling confounding factors using propensity score matching [68].

Compared with inpatients without infection, those with VRE were associated with 1.76 times higher ICU cost [71], and VRE BSI cases were associated with 2.66 times [72] higher total hospital charge and 5.14 times higher total hospital cost [58]. We also found that VRE colonized inpatients led to a significant cost increase of \$1167 than those without colonization after controlling for variables [57] (Table 2 and Additional file 3: Table S2 and S8).

\section{Klebsiella pneumoniae and Escherichia coli}

For both K. pneumoniae and E. coli, cases with extended spectrum $\beta$-lactamases (ESBL)-positive cultures were associated with significantly higher hospital costs or charges compared with ESBL-negative cases [73-75, 80] or those without infection [80]. Direct and indirect cost and total hospital cost for ESBL-positive inpatients was 1.33 times [73] and 1.31-1.72 times [73, 80] as much as that for ESBL-negative cases, respectively. ESBL-positive IAI can attribute to $78 \%$ of increased total hospital cost than ESBL-negative cases [74]. UTI with ESBL-producing bacteria was significantly associated with a 1.52 -fold increase in median hospital cost, 7.58-fold increase in median antibiotic cost [75], and 2.43-fold increase in mean antibiotic cost [76] compared to non-ESBL-producing organisms. $187-200 \%$ of additional hospital cost or charge after culture $[77,78]$ and $66 \%$ of additional total infection cost [79] attributable to ESBL-producing were found [79]. In addition, compared with inpatients without infection, ESBL-positive cases were associated with 3.55-fold total hospital cost [80] (Table 3 and Additional file 3: Table S3 and S8).

For E. coli only, BSI due to third-generation cephalosporins resistance, ESBL-positive, and MDR was associated with 2.12 times, 1.58 times, and 1.28 times of total hospital cost than that for third-generation cephalosporins susceptibility, ESBL-negative, and nonMDR, respectively [36, 82, 90]. Two studies from Thailand explored that community-acquired infection due to ESBL-producing increased hospital costs, with 2.72 times hospital cost after culture than that for non-ESBL-producing, and 4.89 times total hospital 
cost than that for non-infection [81, 83]. The total hospital cost and antibiotic cost of UTI due to resistance to at least one antibiotic (ampicillin, trimethoprim, amoxicillin/ clavulanic acid, cephalexin, ciprofloxacin or nitrofurantoin) and ESBL-producing were considerably higher than that for sensitive to all six antibiotics and non-ESBL-producing after accounting for confounding factors, respectively [84, 85]. Similarly, ESBL-positive colonization was significantly associated with higher total hospital cost and antibiotic cost, with a mean difference of $\$ 1808$ and $\$ 848$, respectively [86]. In addition, the mean difference in total hospital cost and antibiotic cost was $\$ 1574$ and $\$ 81$ between the MDR and non-MDR group in univariate analyses [51]. However, one study reported that there was no significant difference in total hospital cost between ESBL-positive and ESBLnegative BSI after matching for confounders [87] (Table 4 and Additional file 3: Table S4 and S8).

For $K$. pneumoniae only, adjusted median total hospital cost for inpatients with MDR $K$. pneumoniae bloodstream infection was 4.20 time higher than that for non-MDR cases in US [90]. Median costs in total hospital, hospital cost, hospital cost after culture, and antibiotic cost for carbapenem resistant $K$. pneumoniae (CRKP) cases were 1.95 times, 1.33 times, and 2.29 times as high as that for carbapenem susceptible $K$. pneumoniae cases, respectively [89]. One study conducted in China found that the difference in total hospital cost between MDR and non-MDR group was significant [89], while there was no difference in antibiotic cost in a univariate analysis [51] (Table 5 and Additional file 3: Table S5 and S8).

\section{Pseudomonas aeruginosa}

For $P$. aeruginosa, median total hospital cost for inpatients with CRPA was 1.23 times -1.68 times higher than that for those with CSPA after balancing baseline characteristics [91, 92], ranging from $\$ 1065$ in China [91] to $\$ 45,322$ in the US [92]. In univariate analyses, CRPA contributed to 1.51 times of mean hospital cost after culture than CSPA [95], and Eagye et al. found that inpatients with CRPA were associated with 3.09 times median total cost (indirect and direct cost) as high as CSPA cases and 3.91 times higher than those without infection [97]. Resistance and MDR were independently predictive of an increased total hospital cost compared with non-resistance and non-MDR (2.50-fold for resistance vs non-resistance; 3.09 -fold for MDR vs non-resistance; 3.59-fold for MDR vs non-MDR) [51, 94]. [97]. Two studies conducted in the same hospital setting in the US found that resistance to fluoroquinolones and resistance to aztreonam are risk factors for increased total hospital charge and hospital charge after culture, respectively, compared with susceptible cases [93, 96] (Table 6 and Additional file 3: Table S6 and S8).

\section{Acinetobacter baumannii}

For A. baumannii, two studies indicated that inpatients with $C R A B$ were associated with higher total hospital cost and antibiotic cost than CSAB cases after adjusting some confounding factors $[98,99,108]$, and the mean difference in total hospital cost between MDR and nonMDR group ranged from $\$ 6693$ to $\$ 16,074$ in China $[102,103]$. In univariate analyses, mean hospital charge after culture for $C R A B$ cases was 1.21 times -1.65 times higher than that for CSAB cases $[100,108]$, and one study found a significant difference in total hospital cost but not in antibiotic cost among MDR and non-MDR group [51]. Lee et al. explored that MDR colonization was associated with significantly increased ICU cost and total hospital cost [101]. One study in Taiwan, China found significant differences in total hospital cost and antibiotic cost among MDR and non-MDR bacteremia [104]. In addition, MDR inpatients were associated with twice times total hospital charge or cost compared with those without infection [106, 107]. However, there was no significant difference for total hospital cost among infants with ventilator associated pneumonia in the ICU after matching baseline variables between $C R A B$ and CSAB group, and between CRAB and non-infected group [105] (Table 7 and Additional file 3: Table S7 and S8).

\section{Discussion}

ESKAPE species are among the most common bacterial organisms in healthcare-acquired infections, posing a great threat to human health and becoming increasingly more resistant to commonly used antibiotics. This systematic review updates the evidences regarding the economic burden of ABR or MDR ESKAPE organisms compared to susceptible cases or those without infection or colonization. Directing attention towards the ESKAPE organisms can help us to better combat the wide challenge of $A B R$, especially MDR. The studies on the economic cost of $A B R$ are limited chiefly to high-income countries, even though, the current status of ABR may be more serious in the low- and middle-income countries because of scarcity of new medicines, diagnostic tools, and interventions, thus, the value of the economic burden of ABR might be underestimated.

We find that ABR in ESKAPE organisms, is not always, but usually, associated with significantly higher economic burden. In some studies, there are no significant differences in total hospital cost or charge between MRSA and MSSA group [27-36], VRE and VSE group [62, 68], ESBL-positive and ESBL-negative group [87], and CRAB and CSAB group [105]. In addition, the difference in antibiotic cost between MDR and non-MDR group among $A$. baumannii and $E$. coli, the difference in hospital cost before culture between VRE and VSE [69], 
and the difference in hospital cost after culture between MRSA and MSSA [38] have not reached statistical significance as well [51]. The above results may be closely related to study design and patient level factors. Patients with $\mathrm{ABR}$, especially MDR are usually more likely to have more severe illness than those with susceptibility or non-infection, and more likely to be admitted in the ICU, be undergone more surgery, and be taken more antibiotic treatment. It is possible that $A B R$, especially MDR may be associated with higher hospital cost; however, these studies may lack statistical power to detect a significant difference. The results without significant differences are usually drawn after adjustment for confounding variables. If severity of illness is controlled for and all cases are treated similarly, then a cost difference will not be expected. In addition, patients level factors including age (e.g. adult patients, children, or adolescents), source of infection (e.g. BSI, UTI, or IAI), and whether the organism is colonization or infection, are associated with disease status as well, thus may influence the conclusion. Further studies with large sample size, different patients level factors, and controlling confounding factors, are need in the future.

There is one study that even suggested that costs associated with MSSA infection have converged with and may surpass costs of similar MRSA cases [26], which is different compared to historical studies. There are some potential reasons for this diverging result. As mentioned, any study design which controls for severity of illness would be expected to bias the study towards a negative result. It is reported that compared with MSSA, MRSA was associated with a higher mortality rate, thus, we could conclude that it produced a higher severity of illness, which would be expected to require more patient services; however, in this study, patients that died in the hospital and those who were hospitalized for more than 10 days were excluded to eliminate the patients with the most severe infection, and propensity score matching was conducted to reduce the influencing of potential risk factors, which may result that MSSA infections were more severe. Importantly, death is associated with costly economic loss when loss of production and wages are calculated, however, the indirect costs were not considered in this study. MRSA and MSSA infections are treated empirically using vancomycin before the cultures were available. The earlier optimal therapy for MRSA-related infections would improve outcomes and reduce the healthcare cost, however, it is showed a worse outcomes when MSSA-related infections were treated with vancomycin rather than beta-lactam agents [109]. In addition, inpatients with repeated hospitalizations, repeated operations, and repeated infections, which can often cause a prolonged hospital stay with huge costs are not considered in this study. Thus, this findings need to be interpreted with caution.

There is a vast difference in the excess cost among the same comparison groups in the different countries, even within a single country. First, it may be due to the differences between the healthcare systems in the different countries, especially with regard to the medical pricing, insurance system, and reimbursement policy. Second, the different opinions and traditions regarding how to treat infections in different countries are closely associated with the difference in prescribing patterns of antibiotics [110], which further contributes to the geographic differences in ABR [111]. A lot of regional and national surveillance systems have been built to collect representative and accurate ABR data, in order to provide timely information for policy decisions, such as the European Antimicrobial Resistance Surveillance Network (EARS-Net) and Latin American Surveillance Network of Antimicrobial Resistance (ReLAVRA) [17]. WHO launched the Global Antimicrobial Resistance Surveillance System (GLASS) in 2015, which is the first global collaborative and standardized antimicrobial resistance surveillance system [112]. In addition, differences in study design including type of study, study perspective, study method, study population, source of infection or colonization, sample size, and even description of cost likely account for much of the extreme variation in economic outcomes. As is widely known, societal cost for lost productivity for ABR are greater than direct healthcare costs [10], and death may well save healthcare costs but create a severe cost to society and the family in lost wages and production. Some studies developed economic models such as total factor productivity and using a dynamic general equilibrium model to estimate the loss of productivity due to ABR $[14,113]$. Eventually, diverse comparison groups, even in the same bacteria, may result in the differences in comparison of the results in different studies. We find that there is a standard definition for "antibiotic resistance" or "multidrug resistance", but they might not be followed in the different studies, which consequently prevents the public from having a complete comprehension of the extent of the problem of ABR. Policy makers cannot get the accurate information about the rising threat of MDR to public health as well. The European Centre for Disease Prevention and Control (ECDC) and the Centers for Disease Control and Prevention (CDC) created a standardized international terminology to define organisms that are resistant to a significant number of antibiotics. However, it only includes S. aureus, Enterococcus spp., Enterobacteriaceae (other than Salmonella and Shigella), $P$. aeruginosa and Acinetobacter spp., bacteria. Moreover, the same lists of antibiotic categories proposed for antibiotic susceptibility testing in different hospitals, regions, or countries need to be carefully considered [114]. 
The ESKAPE organisms, as a serious global problem, have attached a lot of attention. Recently published research focus on gram-positive bacteria, namely, $S$. aureus and Enterococcus. However, ABR, especially MDR gram-negative bacteria are becoming increasingly prevalent and constitute a serious threat to global public health because they are difficult to treat and are associated with a substantial economic burden $[6,115]$. CRAB, CRPA, and other carbapenem resistant Enterobacteriaceae (CRKP and carbapenem resistant E. coli (CREC)), are classified as priority 1 (critical) on the WHO priority pathogens list for research and development of new antibiotics against ABR [16]. Further studies identifying the effect of resistance on economic outcomes are critical in prioritizing future therapy for these types of bacteria and in optimizing medical resource to control carbapenem resistance.

Our study was subject to certain limitations. First, since we only included articles in the English and Chinese languages, and published literatures, potential language bias and publication bias cannot be neglected. Second, due to different types of values (mean or median) of costs or charges as the primary outcome, no meta-analyses were performed. Third, the majority of studies have been conducted retrospectively; in contrast to a prospective study, there may exist missing data and selection bias due to the retrospective nature [116]. In addition, most of studies were conducted in a single hospital setting, and only took direct cost into consideration regardless of indirect cost, thus, further studies in prospective design, from multiple hospital settings, and on societal cost for ABR are needed. Last, it is limited to ESKAPE organisms chosen because they are among the most important organisms responsible for $\mathrm{ABR}, \mathrm{MDR}$, extensively drug resistance or pan-drug resistance.

\section{Conclusions}

ABR in ESKAPE organisms is not always, but usually, associated with significantly higher economic burden. These results without significant differences may lack statistical power to detect a significant association. Study design which controls for severity of illness and same empirical antibiotic therapy in the two groups would be expected to bias the study towards a similar, even negative result.

There is a vast difference in the excess cost among the same comparison groups in the different countries, even within a single country, which may be due to the different healthcare systems and different opinions and traditions on antibiotic treatments in different countries. Differences in study design and inconsistent standardized definition for ABR and MDR can contribute to diverging results as well.
The review highlights key areas where further researches are needed. Further studies using prospective design, from multiple hospital settings, at a regional and national level are needed. Exploring the loss of production and wages due to $A B R$ or MDR is important for evaluating overall economic burden of ABR. In addition, we should pay more attention to the economic impact of MDR gram-negative bacteria, namely, CRAB, CRPA, CRKP, and CREC.

\section{Additional files}

Additional file 1: Search terms and search strategies. (DOCX $16 \mathrm{~kb}$ )

Additional file 2: Study Quality Assessment. Table S9. Quality assessment checklist for nonrandomized studies. Table S10. Study quality of the included study. (DOCX $120 \mathrm{~kb}$ )

Additional file 3: Table S1. Studies characteristics associated with resistant and multi-drug resistant Staphylococcus aureus. Table S2. Studies

characteristics associated with resistant and multi-drug resistant Enterococcus. Table S3. Studies characteristics associated with resistant and multi-drug resistant E.coli and Klebsiella spp./ K. pneumoniae. Table S4. Studies characteristics associated with resistant and multi-drug resistant E. coli. Table S5. Studies characteristics associated with resistant and multi-drug resistant K. pneumoniae. Table S6. Studies characteristics associated with resistant and multi-drug resistant $P$. aeruginosa. Table $\mathbf{S 7}$. Studies characteristics associated with resistant and multi-drug resistant A. baumannii. Table S8. Studies describing hospital costs among patients with resistant or multi-drug resistant ESKAPE organisms according to different organisms and types of infection. (DOCX $143 \mathrm{~kb})$

\section{Abbreviations}

ABR: Antibiotic resistance; AMR: Antimicrobial resistance; BSI: Bloodstream infection; CDC: Centers for Disease Control and Prevention;

CRAB: Carbapenem resistant A. baumannii; CREC: Carbapenem resistant $E$. coli; CRKP: Carbapenem resistant K. pneumoniae; CRPA: Carbapenem resistant $P$. aeruginosa; CSAB: Carbapenem susceptible A. baumannii;

CSPA: Carbapenem susceptible P. aeruginosa; EARS-Net: Carbapenem susceptible A. baumannii; ECDC: European Centre for Disease Prevention and Control; EEA: European Economic Area; ESBL: Extended spectrum $\beta$ lactamases; EU: European Union; GDP: Gross domestic product; GLASS: Global Antimicrobial Resistance Surveillance System; IAl: Intraabdominal infection; ICU: Intensive care unit; MDR: Multi-drug resistance; MRSA: Methicillin resistant S. aureus; MSSA: Methicillin susceptible S. aureus; NOS: Newcastle-Ottawa quality assessment Scale; ReLAVRA: Latin American Surveillance Network of Antimicrobial Resistance; US: United States; UTI: Urinary tract infection; VRE: Vancomycin resistant Enterococcus; VSE: Vancomycin susceptible Enterococcus; WHO: World Health Organization

\section{Acknowledgements}

It is acknowledged that in the data collection we obtained valuable help from Center for Health Policy Studies, School of Medicine, Zhejiang University.

\section{Authors' contributions}

$\mathrm{XZ}$ participated in the conception and design of this study, performed the searches, independent reviews, data collection, data analysis, and interpretation of data, drafted and revised the manuscript. CSL participated in the conception and design of the study and helped in the revising the manuscript. XS and XH performed the searches, independent reviews, data collection, data analysis, and interpretation of data, drafted and revised the manuscript. HD participated in the conception, design of the study, data collection and interpretation of data, and drafted and revised the manuscript. All authors read and approved the final manuscript.

Funding

This study was supported by China Scholarship Council. 


\section{Availability of data and materials}

All data analysed during this study are provided in the attached file.

\section{Ethics approval and consent to participate}

Not applicable.

\section{Consent for publication}

Not applicable.

\section{Competing interests}

The authors declare that they have no competing interests.

\section{Author details}

${ }^{1}$ Center for Health Policy Studies, School of Public Health, Zhejiang University School of Medicine, 866 Yuhangtang Road, Hangzhou 310058, Zhejiang, China. ${ }^{2}$ Global Health-Health Systems and Policy (HSP): Medicines, focusing antibiotics, Department of Public Health Sciences, Karolinska Institutet, Stockholm, Sweden. ${ }^{3}$ The Fourth Affiliated Hospital Zhejiang University School of Medicine, No. N1, Shancheng Avenue, Yiwu City, Zhejiang, China.

Received: 23 March 2019 Accepted: 31 July 2019

Published online: 13 August 2019

\section{References}

1. World Health Organization. Antibiotic resistance. 2018. https://www.who.int/ news-room/fact-sheets/detail/antibiotic-resistance. Accessed 6 Feb 2018.

2. World Health Organization. Antimicrobial resistance. 2018. https://www.who.int/ news-room/fact-sheets/detail/antimicrobial-resistance. Accessed 14 Feb 2019.

3. Maragakis LL, Perencevich EN, Cosgrove SE. Clinical and economic burden of antimicrobial resistance. Expert Rev Anti-Infect Ther. 2008;6:751-63.

4. Nathwani D, Raman G, Sulham K, Gavaghan M, Menon V. Clinical and economic consequences of hospital-acquired resistant and multidrugresistant Pseudomonas aeruginosa infections: a systematic review and meta-analysis. Antimicrob Resist Infect Control. 2014;3:32.

5. Founou RC, Founou LL, Essack SY. Clinical and economic impact of antibiotic resistance in developing countries: a systematic review and metaanalysis. PLoS One. 2017;12:e189621.

6. Giske CG, Monnet DL, Cars O, Carmeli Y. Clinical and economic impact of common multidrug-resistant gram-negative bacilli. Antimicrob Agents Chemother. 2008;52:813-21.

7. Sipahi OR. Economics of antibiotic resistance. Expert Rev Anti-Infect Ther. 2008;6:523-39.

8. Naylor NR, Atun R, Zhu N, Kulasabanathan K, Silva S, Chatterjee A, et al. Estimating the burden of antimicrobial resistance: a systematic literature review. Antimicrob Resist Infect Control. 2018;7:58.

9. Tansarli GS, Karageorgopoulos DE, Kapaskelis A, Falagas ME. Impact of antimicrobial multidrug resistance on inpatient care cost: an evaluation of the evidence. Expert Rev Anti-Infect Ther. 2013;11:321-31.

10. Centers for Disease Control and Prevention. Antibiotic resistance threats in the United States, 2013. 2013. https://www.cdc.gov/drugresistance/pdf/arthreats-2013-508.pdf. Accessed 6 Feb 2018.

11. European Center for Disease Prevention and Control. The bacterial challenge: time to react. 2009. https://ecdc.europa.eu/sites/portal/files/ media/en/publications/Publications/0909_TER_The_Bacterial_Challenge_ Time_to_React.pdf. Accessed 3 July 2019.

12. European Centers for Disease Control and Prevention. Antimicrobial resistance: trackling the burden in the European Union. 2019. https://www. oecd.org/health/health-systems/AMR-Tackling-the-Burden-in-the-EU-OECDECDC-Briefing-Note-2019.pdf. Accessed 4 July 2019.

13. The World Bank. Drug-resistant infections: a threat to our economic future. 2017. http://documents.worldbank.org/curated/en/323311493396993758/ final-report. Accessed 4 July 2018.

14. RAND Corporation. Estimating the economic costs of antimicrobial resistance. 2014. https://www.rand.org/randeurope/research/projects/ antimicrobial-resistance-costs.html. Accessed 2 Dec 2018.

15. World Health Organization. Global action plan on antimicrobial resistance. 2015. https://apps.who.int/iris/bitstream/handle/10665/193736/97892415 09763_eng.pdf?sequence=1. Accessed 12 Feb 2019.

16. World Health Organization. Global priority list of antibiotic resistant bacteria to guide research, discovery, and development of new antibiotics. https://
www.who.int/medicines/publications/WHO-PPL-Short_Summary_25Feb-ET_ NM_WHO.pdf. Accessed 6 Feb 2019.

17. World Health Organization. Antimicrobial resistance global report on surveillance. 2014. https://www.who.int/drugresistance/documents/ surveillancereport/en/. Accessed 11 Dec 2018.

18. Santajit S, Indrawattana N. Mechanisms of antimicrobial resistance in ESKAPE pathogens. Biomed Res Int. 2016;2016:2475067.

19. Pendleton JN, Gorman SP, Gilmore BF. Clinical relevance of the ESKAPE pathogens. Expert Rev Anti-Infect Ther. 2013;11:297-308.

20. Pogue JM, Kaye KS, Cohen DA, Marchaim D. Appropriate antimicrobial therapy in the era of multidrug-resistant human pathogens. Clin Microbiol Infect. 2015;21:302-12.

21. Bodro M, Sabe N, Tubau F, Llado L, Baliellas C, Roca J, et al. Risk factors and outcomes of bacteremia caused by drug-resistant ESKAPE pathogens in solid-organ transplant recipients. Transplantation. 2013;96:843-9.

22. Karlowsky JA, Hoban DJ, Hackel MA, Lob SH, Sahm DF. Resistance among gram-negative ESKAPE pathogens isolated from hospitalized patients with intra-abdominal and urinary tract infections in Latin American countries: SMART 2013-2015. Braz J Infect Dis. 2017;21:343-8.

23. Karlowsky JA, Hoban DJ, Hackel MA, Lob SH, Sahm DF. Antimicrobial susceptibility of gram-negative ESKAPE pathogens isolated from hospitalized patients with intra-abdominal and urinary tract infections in Asia-Pacific countries: SMART 2013-2015. J Med Microbiol. 2017;66:61-9.

24. Organisation for Economic Cooperation and Development. Exchange rates, end of period. 2019. https://stats.oecd.org/index.aspx?queryid=221\#. Accessed 15 Feb 2019.

25. Organisation for Economic Cooperation and Development. Consumer price indices. 2019. https://stats.oecd.org/index.aspx?queryid=221\#. Accessed 15 Feb 2019.

26. Klein EY, Jiang W, Mojica N, Tseng KK, McNeill R, Cosgrove SE, et al. National costs associated with methicillin-susceptible and methicillin-resistant Staphylococcus aureus hospitalizations in the United States, 2010-2014. Clin Infect Dis. 2019;68:22-8.

27. Chen W, Li S, Li H, Zhang S, Liu B, Zhang X, et al. Comparison in prognosis of hospital-acquired pneumonia due to methicillin-resistant and methicillinsensitive Staphylococcus aureus: analysis of propensity score matching. Chin J Infect Control. 2016;15:299-303.

28. Shorr AF, Haque N, Taneja C, Zervos M, Lamerato L, Kothari S, et al. Clinical and economic outcomes for patients with health care-associated Staphylococcus aureus pneumonia. J Clin Microbiol. 2010;48:3258-62.

29. Taneja C, Haque N, Oster G, Shorr AF, Zilber S, Kyan PO, et al. Clinical and economic outcomes in patients with community-acquired Staphylococcus aureus pneumonia. J Hosp Med. 2010;5:528-34.

30. Shorr AF, Tabak YP, Gupta V, Johannes RS, Liu LZ, Kollef MH. Morbidity and cost burden of methicillin-resistant Staphylococcus aureus in early onset ventilator-associated pneumonia. Crit care (London, England). 2006;10:R97.

31. Itani KM, Merchant S, Lin SJ, Akhras K, Alandete JC, Hatoum HT. Outcomes and management costs in patients hospitalized for skin and skin-structure infections. Am J Infect Control. 2011;39:42-9.

32. Li X, Chen Y, Gao W, Ouyang W, Wei J, Wen Z. Epidemiology and outcomes of complicated skin and soft tissue infections among inpatients in southern China from 2008 to 2013. PLoS One. 2016;11:e149960.

33. Park SY, Son JS, Oh $H$, Choi JM, Lee MS. Clinical impact of methicillinresistant Staphylococcus aureus bacteremia based on propensity scores. Infection. 2011;39:141-7.

34. Branch-Elliman W, Lee GM, Golen TH, Gold HS, Baldini LM, Wright SB. Health and economic burden of post-partum Staphylococcus aureus breast abscess. PLoS One. 2013;8:e73155.

35. Kopp BJ, Nix DE, Armstrong EP. Clinical and economic analysis of methicillin-susceptible and -resistant Staphylococcus aureus infections. Ann Pharmacother. 2004;38:1377-82.

36. de Kraker MEA, Davey PG, Grundmann H. Mortality and hospital stay associated with resistant Staphylococcus aureus and Escherichia coli bacteremia: estimating the burden of antibiotic resistance in Europe. PLoS Med. 2011;8:e1001104

37. Ott E, Bange FC, Reichardt C, Graf K, Eckstein M, Schwab F, et al. Costs of nosocomial pneumonia caused by meticillin-resistant Staphylococcus aureus. J Hosp Infect. 2010;76:300-3.

38. Ben-David D, Novikov I, Mermel LA. Are there differences in hospital cost between patients with nosocomial methicillin-resistant Staphylococcus aureus 
bloodstream infection and those with methicillin-susceptible S. aureus bloodstream infection? Infect Control Hosp Epidemiol. 2009:30:453-60.

39. McHugh CG, Riley LW. Risk factors and costs associated with methicillinresistant Staphylococcus aureus bloodstream infections. Infect Control Hosp Epidemiol. 2004;25:425-30.

40. Thampi N, Showler A, Burry L, Bai AD, Steinberg M, Ricciuto DR, et al. Multicenter study of health care cost of patients admitted to hospital with Staphylococcus aureus bacteremia: impact of length of stay and intensity of care. Am J Infect Control. 2015;43:739-44.

41. Rubio-Terres C, Garau J, Grau S, Martinez-Martinez L. Cost of bacteraemia caused by methicillin-resistant vs. methicillin-susceptible Staphylococcus aureus in Spain: a retrospective cohort study. Clin Microbiol Infect. 2010;16:722-8.

42. Reed SD, Friedman JY, Engemann JJ, Griffiths RI, Anstrom KJ, Kaye KS, et al. Costs and outcomes among hemodialysis-dependent patients with methicillin-resistant or methicillin-susceptible Staphylococcus aureus bacteremia. Infect Control Hosp Epidemiol. 2005;26:175-83.

43. Engemann JJ, Carmeli Y, Cosgrove SE, Fowler VG, Bronstein MZ, Trivette SL, et al. Adverse clinical and economic outcomes attributable to methicillin resistance among patients with Staphylococcus aureus surgical site infection. Clin Infect Dis. 2003;36:592-8.

44. Anderson DJ, Kaye KS, Chen LF, Schmader KE, Choi Y, Sloane R, et al. Clinical and financial outcomes due to methicillin resistant Staphylococcus aureus surgical site infection: a multi-center matched outcomes study. PLoS One. 2009;4:e8305.

45. Song X, Perencevich E, Campos J, Short BL, Singh N. Clinical and economic impact of methicillin-resistant Staphylococcus aureus colonization or infection on neonates in intensive care units. Infect Control Hosp Epidemiol. 2010;31:177-82

46. Filice GA, Nyman JA, Lexau C, Lees CH, Bockstedt LA, Como-Sabetti K, et al. Excess costs and utilization associated with methicillin resistance for patients with Staphylococcus aureus infection. Infect Control Hosp Epidemiol. 2010;31:365-73.

47. Nelson RE, Jones M, Liu CF, Samore MH, Evans ME, Graves N, et al. The impact of healthcare-associated methicillin-resistant Staphylococcus aureus infections on post-discharge healthcare costs and utilization. Infect Control Hosp Epidemiol. 2015;36:534-42.

48. Lee YJ, Chen JZ, Lin HC, Liu HY, Lin SY, Lin HH, et al. Impact of active screening for methicillin-resistant Staphylococcus aureus (MRSA) and decolonization on MRSA infections, mortality and medical cost: a quasiexperimental study in surgical intensive care unit. Crit Care (London, England). 2015;19:143.

49. Resch A, Wilke M, Fink C. The cost of resistance: incremental cost of methicillin-resistant Staphylococcus aureus (MRSA) in German hospitals. Eur J Health Econ. 2009;10:287-97.

50. Nelson RE, Samore MH, Jones M, Greene T, Stevens WW, Liu CF, et al. Reducing time-dependent bias in estimates of the attributable cost of health care-associated methicillin-resistant Staphylococcus aureus infections: a comparison of three estimation strategies. Med Care. 2015;53:827-34.

51. Xu B, Yuan H, Yang P. Evaluation of economic burden induced by multidrug-resistant bacteria related infections in a tertiary general hospital. Chinese J Exp Clin Infect Dis. 2017;11:455-9.

52. Capitano B, Leshem OA, Nightingale CH, Nicolau DP. Cost effect of managing methicillin-resistant Staphylococcus aureus in a long-term care facility. J Am Geriatr Soc. 2003;51:10-6.

53. Cosgrove SE, Qi Y, Kaye KS, Harbarth S, Karchmer AW, Carmeli Y. The impact of methicillin resistance in Staphylococcus aureus bacteremia on patient outcomes: mortality, length of stay, and hospital charges. Infect Control Hosp Epidemiol. 2005;26:166-74.

54. Lodise TP, McKinnon PS. Clinical and economic impact of methicillin resistance in patients with Staphylococcus aureus bacteremia. Diagn Microbiol Infect Dis. 2005;52:113-22.

55. Kim CJ, Kim HB, Oh MD, Kim Y, Kim A, Oh SH, et al. The burden of nosocomial Staphylococcus aureus bloodstream infection in South Korea: a prospective hospital-based nationwide study. BMC Infect Dis. 2014;14:590.

56. Fu J, Chen B, Wang X, Ye Q, Lu Z, Su C, et al. Case-control study of influence of methicillin-resistant Staphylococcus aureus infection on economic losses and length of hospital stay. Chin J Nosocomiol. 2014;24: 2363-5

57. Engler-Huesch S, Heister T, Mutters NT, Wolff J, Kaier K. In-hospital costs of community-acquired colonization with multidrug-resistant organisms at a German teaching hospital. BMC Health Serv Res. 2018;18:737.
58. Butler AM, Olsen MA, Merz LR, Guth RM, Woeltje KF, Camins BC, et al. Attributable costs of Enterococcal bloodstream infections in a nonsurgical hospital cohort. Infect Control Hosp Epidemiol. 2010;31:28-35.

59. Ford CD, Lopansri BK, Haydoura S, Snow G, Dascomb KK, Asch J, et al. Frequency, risk factors, and outcomes of vancomycin-resistant Enterococcus colonization and infection in patients with newly diagnosed acute leukemia: different patterns in patients with acute myelogenous and acute lymphoblastic leukemia. Infect Control Hosp Epidemiol. 2015;36:47-53.

60. Kramer TS, Remschmidt C, Werner S, Behnke M, Schwab F, Werner G, et al. The importance of adjusting for enterococcus species when assessing the burden of vancomycin resistance: a cohort study including over 1000 cases of enterococcal bloodstream infections. Antimicrob Resist Infect Control. 2018;7:133.

61. Cheah AL, Spelman T, Liew D, Peel T, Howden BP, Spelman D, et al. Enterococcal bacteraemia: factors influencing mortality, length of stay and costs of hospitalization. Clin Microbiol Infect. 2013;19:E181-9.

62. Lloyd-Smith P, Younger J, Lloyd-Smith E, Green H, Leung V, Romney MG. Economic analysis of vancomycin-resistant enterococci at a Canadian hospital: assessing attributable cost and length of stay. J Hosp Infect. 2013;85:54-9.

63. Adams DJ, Eberly MD, Goudie A, Nylund CM. Rising vancomycin-resistant Enterococcus infections in hospitalized children in the United States. Hosp Pediatr. 2016;6:404-11

64. Gearhart M, Martin J, Rudich S, Thomas M, Wetzel D, Solomkin J, et al. Consequences of vancomycin-resistant Enterococcus in liver transplant recipients: a matched control study. Clin Transpl. 2005;19:711-6.

65. Webb M, Riley LW, Roberts RB. Cost of hospitalization for and risk factors associated with vancomycin-resistant Enterococcus faecium infection and colonization. Clin Infect Dis. 2001;33:445-52.

66. Carmeli Y, Eliopoulos G, Mozaffari E, Samore M. Health and economic outcomes of vancomycin-resistant enterococci. Arch Intern Med. 2002;162:2223-8.

67. Nguyen GC, Leung W, Weizman AV. Increased risk of vancomycin-resistant Enterococcus (VRE) infection among patients hospitalized for inflammatory bowel disease in the United States. Inflamm Bowel Dis. 2011;17:1338-42.

68. Jung E, Byun S, Lee H, Moon SY, Lee H. Vancomycin-resistant Enterococcus colonization in the intensive care unit: clinical outcomes and attributable costs of hospitalization. Am J Infect Control. 2014:42:1062-6.

69. Puchter L, Chaberny IF, Schwab F, Vonberg RP, Bange FC, Ebadi E. Economic burden of nosocomial infections caused by vancomycin-resistant enterococci. Antimicrob Resist Infect Control. 2018;7:1.

70. Jiang $\mathrm{HL}$, Zhou Z, Wang LS, Fang Y, Li YH, Chu Cl. The risk factors, costs, and survival analysis of invasive VRE infections at a medical center in eastern Taiwan. Int J Infect Dis. 2017;54:18-24.

71. Pelz RK, Lipsett PA, Swoboda SM, Diener-West M, Powe NR, Brower RG, et al. Vancomycin-sensitive and vancomycin-resistant enterococcal infections in the ICU: attributable costs and outcomes. Intensive Care Med. 2002;28:692-7.

72. Song XY, Srinivasan A, Plaut D, Perl TM. Effect of nosocomial vancomycin-resistant enterococcal bacteremia on mortality, length of stay, and costs. Infect Control Hosp Epidemiol. 2003;24:251-6.

73. Maslikowska JA, Walker SAN, Elligsen M, Mittmann N, Palmay L, Daneman N, et al. Impact of infection with extended-spectrum beta-lactamaseproducing Escherichia coli or Klebsiella species on outcome and hospitalization costs. J Hosp Infect. 2016;92:33-41.

74. Hu B, Ye H, Xu Y, Ni Y, Hu Y, Yu Y, et al. Clinical and economic outcomes associated with community-acquired intra-abdominal infections caused by extended spectrum beta-lactamase (ESBL) producing bacteria in China. Curr Med Res Opin. 2010;26:1443-9.

75. MacVane SH, Tuttle LO, Nicolau DP. Impact of extended-spectrum beta-lactamase-producing organisms on clinical and economic outcomes in patients with urinary tract infection. J Hosp Med. 2014;9:232-8.

76. Yang Y, Ku C, Lin J, Shang S, Chiu C, Yeh K, et al. Impact of extendedspectrum beta-lactamase-producing Escherichia coli and Klebsiella pneumoniae on the outcome of community-onset bacteremic urinary tract infections. J Microbiol Immunol Infect. 2010;43:194-9.

77. Apisarnthanarak A, Kiratisin P, Mundy LM. Predictors of mortality from community-onset bloodstream infections due to extended-spectrum beta-lactamase-producing Escherichia coli and Klebsiella pneumoniae. Infect Control Hosp Epidemiol. 2008;29:671-4.

78. Lautenbach E, Patel JB, Bilker WB, Edelstein PH, Fishman NO. Extendedspectrum beta-lactamase-producing Escherichia coli and Klebsiella pneumoniae: risk factors for infection and impact of resistance on outcomes. Clin Infect Dis. 2001;32:1162-71. 
79. Lee SY, Kotapati S, Kuti JL, Nightingale CH, Nicolau DP. Impact of extendedspectrum beta-lactamase-producing Escherichia coli and Klebsiella species on clinical outcomes and hospital costs: a matched cohort study. Infect Control Hosp Epidemiol. 2006;27:1226-32.

80. Apisarnthanarak A, Kiratisin P, Saifon P, Kitphati R, Dejsirilert S, Mundy LM. Risk factors for and outcomes of healthcare-associated infection due to extended-spectrum beta-lactamase-producing Escherichia coli or Klebsiella pneumoniae in Thailand. Infect Control Hosp Epidemiol. 2007;28:873-6.

81. Tumbarello M, Spanu T, Di Bidino R, Marchetti M, Ruggeri M, Trecarichi EM, et al. Costs of bloodstream infections caused by Escherichia coli and influence of extended-spectrum-beta-lactamase production and inadequate initial antibiotic therapy. Antimicrob Agents Chemother. 2010;54:4085-91.

82. Thaden JT, Li Y, Ruffin F, Maskarinec SA, Hill-Rorie JM, Wanda LC, et al. Increased costs associated with bloodstream infections caused by multidrugresistant gram-negative bacteria are due primarily to patients with hospitalacquired infections. Antimicrob Agents Chemother. 2017;61:e01709-16.

83. Apisarnthanarak A, Kiratisin P, Saifon P, Kitphati R, Dejsirilert S, Mundy LM. Predictors of mortality among patients with community-onset infection due to extended-spectrum beta-lactamase producing Escherichia coli in Thailand. Infect Control Hosp Epidemiol. 2008;29:80-2.

84. Apisarnthanarak A, Kiratisin P, Saifon P, Kitphati R, Dejsirilert S, Mundy LM. Clinical and molecular epidemiology of community-onset, extendedspectrum beta-lactamase-producing Escherichia coli infections in Thailand: a case-case-control study. Am J Infect Control. 2007;35:606-12.

85. Alam MF, Cohen D, Butler C, Dunstan F, Roberts Z, Hillier S, et al. The additional costs of antibiotics and re-consultations for antibiotic-resistant Escherichia coli urinary tract infections managed in general practice. Int J Antimicrob Agents. 2009;33:255-7.

86. Esteve-Palau E, Solande G, Sanchez F, Sorli L, Montero M, Gueerri R, et al. Clinical and economic impact of urinary tract infections caused by ESBLproducing Escherichia coli requiring hospitalization: a matched cohort study. J Infect. 2015;71:667-74

87. Cornejo-Juarez P, Suarez-Cuenca JA, Volkow-Fernandez P, Silva-Sanchez J, Barrios-Camacho H, Najera-Leon E, et al. Fecal ESBL Escherichia coli carriage as a risk factor for bacteremia in patients with hematological malignancies. Support Care Cancer. 2016;24:253-9.

88. Meng X, Liu S, Duan J, Huang X, Zhou P, Xiong X, et al. Risk factors and medical costs for healthcare-associated carbapenem-resistant Escherichia coli infection among hospitalized patients in a Chinese teaching hospital. BMC Infect Dis. 2017;17:82.

89. Leistner R, Bloch A, Sakellariou C, Gastmeier P, Schwab F. Costs and length of stay associated with extended-spectrum $\beta$-lactamase production in cases of Escherichia coli bloodstream infection. J Glob Antimicrob Resist. 2014;2: 107-9.

90. Huang W, Qiao F, Zhang Y, Huang J, Deng Y, Li J, et al. In-hospital medical costs of infections caused by carbapenem-resistant Klebsiella pneumoniae. Clin Infect Dis. 2018:672:S225-30

91. Chen Z, Xu Z, Wu H, Chen L, Gao S, Chen Y. The impact of carbapenemresistant Pseudomonas aeruginosa on clinical and economic outcomes in a Chinese tertiary care hospital: a propensity score-matched analysis. Am J Infect Control. 2018;47:677-82.

92. Lautenbach E, Weiner MG, Nachamkin I, Bilker WB, Sheridan A, Fishman NO. Imipenem resistance among pseudomonas aeruginosa isolates: risk factors for infection and impact of resistance on clinical and economic outcomes. Infect Control Hosp Epidemiol. 2006;27:893-900.

93. Gasink LB, Fishman NO, Weiner MG, Nachamkin I, Bilker WB, Lautenbach E. Fluoroquinolone-resistant Pseudomonas aeruginosa: assessment of risk factors and clinical impact. Am J Med. 2006;119:526-e19.

94. Morales E, Cots F, Sala M, Comas M, Belvis F, Riu M, et al. Hospital costs of nosocomial multi-drug resistant Pseudomonas aeruginosa acquisition. BMC Health Serv Res. 2012;12:122.

95. Lautenbach E, Synnestvedt M, Weiner MG, Bilker WB, Vo L, Schein J, et al. Imipenem resistance in Pseudomonas aeruginosa: emergence, epidemiology, and impact on clinical and economic outcomes. Infect Control Hosp Epidemiol. 2010;31:47-53.

96. Gasink LB, Fishman NO, Nachamkin I, Bilker WB, Lautenbach E. Risk factors for and impact of infection or colonization with aztreonam-resistant Pseudomonas aeruginosa. Infect Control Hosp Epidemiol. 2007;28:1175-80.

97. Eagye KJ, Kuti JL, Nicolau DP. Risk factors and outcomes associated with isolation of meropenem high-level-resistant Pseudomonas aeruginosa. Infect Control Hosp Epidemiol. 2009;30:746-52.
98. Cui N, Cao B, Liu Y, Liang L, Gu L, Song S. The impact of imipenem-resistant Acinetobacter baumannii infection on clinical outcomes and medical care costs. Chin J Infect Dis. 2012;30:209-14.

99. Zhen X, Chen Y, Hu X, Dong P, Gu S, Sheng YY, et al. The difference in medical costs between carbapenem-resistant Acinetobacter baumannii and non-resistant groups: a case study from a hospital in Zhejiang province, China. Eur J Clin Microbiol Infect Dis. 2017;36:1989-94.

100. Lautenbach E, Synnestvedt M, Weiner MG, Bilker WB, Vo L, Schein J, et al. Epidemiology and impact of imipenem resistance in Acinetobacter baumannii. Infect Control Hosp Epidemiol. 2009;30:1186-92.

101. Lee $\mathrm{H}$, Lee $\mathrm{H}$. Clinical and economic evaluation of multidrug-resistant Acinetobacter baumannii colonization in the intensive care unit. Infect Chemother. 2016:48:174-80.

102. Wu X, Ding L, Wu X. Direct economic loss due to healthcare-associated infection with multidrug resistant Acinetobacter baumannii. Chin J Infect Control. 2018;17:735-8.

103. Guo Y, Guo W, Qiu P, He Q, Pan C, Wu C, et al. Study of attributive hospitalized cost and length of stay for hospital acquired infection due to multidrug resistance and none-resistance Acinetobacter baumannii. Chin J Health Stat. 2017:34:378-81.

104. Lee NY, Lee HC, Ko NY, Chang CM, Shih HI, Wu CJ, et al. Clinical and economic impact of multidrug resistance in nosocomial Acinetobacter baumannii bacteremia. Infect Control Hosp Epidemiol. 2007;28:713-9.

105. Thatrimontrichai A, Techato C, Dissaneevate S, Janjindamai W, Maneenil G, Kritsaneepaiboon S, et al. Risk factors and outcomes of carbapenemresistant Acinetobacter baumannii ventilator-associated pneumonia in the neonate: a case-case-control study. J Infect Chemother. 2016;22:444-9.

106. Young LS, Sabel AL, Price CS. Epidemiologic, clinical, and economic evaluation of an outbreak of clonal multidrug-resistant Acinetobacter baumannii infection in a surgical intensive care unit. Infect Control Hosp Epidemiol. 2007;28:1247-54.

107. Wilson SJ, Knipe CJ, Zieger MJ, Gabehart KM, Goodman JE, Volk HM, et al. Direct costs of multidrug-resistant Acinetobacter baumannii in the burn unit of a public teaching hospital. Am J Infect Control. 2004;32:342-4.

108. Lemos EV, de la Hoz FP, Alvis N, Einarson TR, Quevedo E, Castaneda C, et al. Impact of carbapenem resistance on clinical and economic outcomes among patients with Acinetobacter baumannii infection in Colombia. Clin Microbiol Infect. 2014;20:174-80.

109. McDanel JS, Perencevich EN, Diekema DJ, Herwaldt LA, Smith TC, Chrischilles EA, et al. Comparative effectiveness of beta-lactams versus vancomycin for treatment of methicillin-susceptible Staphylococcus aureus bloodstream infections among 122 hospitals. Clin Infect Dis. 2015;61:361-7.

110. Llor C, Bjerrum L. Background for different use of antibiotics in different countries. Clin Infect Dis. 2005:40:333.

111. Asbell PA, Pandit RT, Sanfilippo CM. Antibiotic resistance rates by geographic region among ocular pathogens collected during the ARMOR surveillance study. Ophthalmol Therapy. 2018;7:417-29.

112. World Health Organization. Global antimicrobial resistance surveillance system (GLASS) report-early implementation 2016-2017. 2018. https://www. who.int/glass/resources/publications/early-implementation-report/en/. Accessed 5 Mar 2019.

113. KPMG. The economic impact of anti-microbial resistance. 2014. https:// home.kpmg.com/content/dam/kpmg/pdf/2014/12/amr-report-final.pdf. Accessed 2 Dec 2018.

114. Magiorakos AP, Srinivasan A, Carey RB, Carmeli Y, Falagas ME, Giske CG, et al. Multidrug-resistant, extensively drug-resistant and pandrug-resistant bacteria: an international expert proposal for interim standard definitions for acquired resistance. Clin Microbiol Infect. 2012;18:268-81.

115. Kaye KS, Pogue JM. Infections caused by resistant gram-negative Bacteria: epidemiology and management. Pharmacotherapy. 2015;35:949-62.

116. Weinger MB, Slagle J, Jain S, Ordonez N. Retrospective data collection and analytical techniques for patient safety studies. J Biomed Inform. 2003;36: 106-19.

\section{Publisher's Note}

Springer Nature remains neutral with regard to jurisdictional claims in published maps and institutional affiliations. 